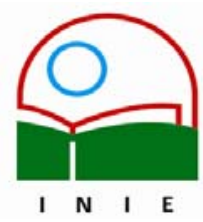

Actualidades Investigativas en Educación

Revista Electrónica publicada por el

Instituto de Investigación en Educación

Universidad de Costa Rica

ISSN 1409-4703

http://revista.inie.ucr.ac.cr

COSTA RICA

\title{
RETOS ACTUALES DE LA INVESTIGACIÓN EDUCATIVA
}

CURRENT CHALLENGES OF THE EDUCATIONAL RESEARCH

\author{
Volumen 8 , Número 3
}

pp. 1-18

Este número se publicó el 15 de diciembre 2008

Flor Delgado de Colmenares

La revista está indexada en los directorios:

LATINDEX, REDALYC, IRESIE, CLASE, DIALNET, DOAJ, E-REVIST@S,

La revista está incluida en los sitios:

REDIE, RINACE, OEI, MAESTROTECA, HUASCARAN

Los contenidos de este artículo están bajo una licencia Creative Commons 


\title{
RETOS ACTUALES DE LA INVESTIGACIÓN EDUCATIVA CURRENT CHALLENGES OF THE EDUCATIONAL RESEARCH
}

\section{Flor Delgado de Colmenares ${ }^{1}$}

\begin{abstract}
Resumen: El presente artículo constituye parte de los resultados de una investigación ya concluida, donde se realizó un estudio crítico del estado de la investigación educativa en general y su práctica tradicional en la escuela latinoamericana; se trató de una investigación de tipo analítica e interpretativa - crítica de la obra de autores seleccionados y de experiencias practicas llevadas a cabo. El artículo en cuestión tiene como propósito contribuir a la discusión y reflexión sobre este importante tema, en el marco de la crisis de paradigmas de las ciencias sociales y frente a la asunción de nuevas perspectivas teóricas que tienen entre sus premisas la reivindicación de lo humano y cualitativo en la búsqueda del conocimiento de lo social y educativo.
\end{abstract}

Palabras clave: INVESTIGACIÓN EDUCATIVA, PARADIGMAS, PERSPECTIVAS CUALITATIVAS.

\begin{abstract}
The present article is part of the results of a research, a critical study of the state of the educational research and the traditional practice of the same one in the Latin American. The article in question has as purpose to contribute to the discussion and reflection on this important topic, in the mark of the crisis of paradigms of the social sciences and in front of the assumption of theoretical new perspectives that you/they have among its premises the recovery of the human and qualitative in the search of the knowledge of the social and educational.
\end{abstract}

Key words: EDUCATIONAL INVESTIGATION, PARADIGM ESTABLISHED, QUALITATIVE PERSPECTIVES.

\section{PRESENTACIÓN}

Acercarse con una visión crítica al estado actual de la investigación educativa implica reconocer que la manera como ésta es asumida y practicada, lleva en sí un carácter valorativo que guarda estrecha relación con la concepción doctrinaria de la que ella se deriva. Es decir, que su dirección y orientación obedece a la concepción de la educación y del ser humano que rige el deber ser de los proyectos educativos y curriculares establecidos en las escuelas de educación de las universidades públicas latinoamericanas.

\footnotetext{
1 Doctora en Ciencias Humanas; Coordinadora de la Linea de Investigación: Alternativas cualitativas en educación y su investigación, Profesora investigadora de la Universidad de los Andes en Trujillo, Venezuela.
}

Dirección electrónica: flordelba@yahoo.es

Artículo recibido: 18 de febrero, 2008

Aprobado: 10 de noviembre, 2008 
Es por ello, que ante los retos del presente cualquier reflexión en torno a la investigación educativa debe comenzar por la revisión de las concepciones que subyacen en la conjunción con la investigación en ciencias sociales y en el marco de los paradigmas vigentes. En tal sentido, se postula que la investigación educativa tradicional está condicionada por la concepción positivista de la educación y de la sociedad, en la que se imponen teorías y metodologías que predeterminan la realidad contextual objeto de investigación y la forma cómo abordarla.

Por estas razones, en las perspectivas emergentes de tendencia humanista, al referirse a la investigación educativa, algunos autores, entre ellos, Colom (2004), Morin (2002), coinciden en afirmar que existe urgencia de un replanteo epistemológico y teórico de la concepción y práctica tradicional de la misma por la poca o ninguna incidencia de los resultados en la toma de conciencia de la necesidad de reivindicar lo humano desde un plano crítico y comprometido a partir de la educación.

Tal situación anima la presentación de este artículo con el propósito de propiciar la discusión y reflexión acerca del estado actual de la investigación educativa y las posibilidades de transformación en función de las nuevas propuestas paradigmáticas con base en la comprensión de lo socioeducativo desde lo humano y cualitativo.

\section{EL ESCENARIO DE LA INVESTIGACIÓN EDUCATIVA}

La investigación educativa ha recorrido un camino marcado por el positivismo como paradigma social; desde la concepción de ésta en el marco de la pedagogía experimental hasta ser utilizada como un instrumento para la reproducción del conocimiento socialmente establecido; lo que ha conducido, en la actualidad, a la justificación de lo económico y tecnológico, como la única posibilidad de construcción y recreación de todo lo social y humano. Una teoría y práctica de investigación que han conducido a la profundización de nociones de objetividad, racionalidad y verdad en las que el conocimiento tiene un papel instrumental en la solución de los problemas educacionales y, en las cuales, los problemas sociales y educativos se han restringido a explicaciones desde el punto de vista técnico, estadístico y cuantitativo. 
Hoy día, la investigación social y educativa de carácter positivista es objeto de serios cuestionamientos por parte de una visión emergente crítica de las ciencias sociales (Habermas, Morin), en la cual se cuestiona, entre otros aspectos, el reduccionismo de lo humano, razón por la cual no ha sido posible explicar a cabalidad las diversas situaciones del complejo contexto socio educativo; y, lo restrictivo del método que limita el abordaje de lo complejo e interdisciplinario de los diversos escenarios y problemas humanos y sociales contemporáneos.

Entre los postulados críticos a la concepción establecida de investigación educativa, destacan las tendencias de carácter fenomenológico crítico, las cuales coinciden en la necesidad del retorno del sujeto y la revitalización de lo humano, presente en los procesos sociales y educativos abordados en el marco de una perspectiva histórico cultural social, cuyo centro de interés sea la persona; su vida cotidiana; sus creencias; simbolismos, prácticas y, el significado que éstas tienen para el entendimiento de lo socioeducativo (Ávila, 2005).

En esta encrucijada se encuentran las ciencias sociales y humanas, con la asunción de nuevos paradigmas que demandan un giro cualitativo en el tratamiento de lo social frente al imperio del objetivismo e instrumentalismo, como única vía para la búsqueda del conocimiento. De allí que, tal como lo plantea Delgado (2001, p. 12), acercarse con una visión crítica al estado actual de la investigación educativa implica reconocer que la manera como ésta es asumida y practicada, lleva en sí un carácter valorativo que guarda estrecha relación con la concepción doctrinaria de la que ella se deriva.

En tal sentido, en los postulados actuales sobre la investigación educativa, existe coincidencia, desde la crítica social, en relación a la urgencia de un replanteo de los paradigmas teóricos tradicionales y sus metodologías de investigación, en especial, en la toma de conciencia de la necesidad de reivindicar lo humano desde un plano crítico y comprometido a partir de la educación, planteamiento que se reafirma cuando Esqueda expresa:

La necesidad de una reconstrucción teórica y el escepticismo acerca de la significación social de la investigación educativa no implica, la ausencia de problemas que deban ser investigados o un debilitamiento de la capacidad investigativa de los científicos de 
la educación. Las carencias en el conocimiento son significativas y numerosa la actividad de investigación pero, a pesar de ser ésta muy intensa, aún así no acaba de completar el vacío teórico y práctico existente debido a la existencia de un componente normativo y epistemológico en el fondo de esta situación que generalmente se obvia... (2004, p. 76)

Lo anterior indica que la investigación educativa no es una actividad neutra sino que lleva una intencionalidad, lo que requiere de la sensibilidad y de la toma de conciencia por parte de los actores del proceso para identificar y cuestionar aquellos aspectos que desvirtúan la razón de ser de la realidad práctica de la investigación.

En tanto, ya es voz común el decir que el paradigma teórico dominante, es decir el positivismo, en las últimas décadas, está mostrando evidentes síntomas de agotamiento en su capacidad explicativa. La teoría educativa, en consecuencia, enfrenta un problema de producción debido a que los aspectos más significativos o particulares de la realidad socioeducativa contemporánea quedan fueran de su alcance. Lo planteado, caracteriza una práctica educativa formalizada en la cual subyace una concepción particular de la educación y de la sociedad, delineada en la filosofía de los proyectos educativos, por lo que es preciso adentrarse en el contexto de la educación actual para reconocer y comprender las diferentes prácticas y procesos que en ella se realizan.

Al analizar lo planteado por Morin (2002), se infiere que entre las características de la educación tradicional destacan: la concepción del ser humano como objeto de la educación y la educación formal, como un proceso dirigido al logro de mayor productividad mediante el dominio de la técnica; legitimando, cada vez más la tesis de la formación del capital humano para el desarrollo económico. De esta manera, los criterios que subyacen en la planificación de la actividad educativa e investigativa dan razón del diseño y ejecución de programas de investigación de corte experimentalista y causal, en la búsqueda de la eficiencia de lo educativo hacia la modernización social. Presupuestos teóricos y prácticos que han ido facilitando un proceso educativo que anula la capacidad crítica de los individuos y la falta de identidad y compromiso para con lo social: un ser ahistórico, desarraigado y desidentificado. 
En tal sentido, al contextualizar la situación anteriormente planteada en el marco de la crisis actual de la educación, puede decirse que esta crisis es una crisis humana. Situación que no es reconocida como tal por los entes oficiales encargados de darle soluciones, ya que es percibida y tratada como un aspecto que debe ser reformado más no transformado, provocando, por tanto, un alto nivel de concentración en los esfuerzos que se realizan y una baja capacidad de resolución y de pertinencia en los conocimientos que se producen.

De esta forma, los resultados generados en el marco del paradigma establecido no agregan elementos significativamente novedosos al conocimiento ya acumulado, y las investigaciones que se apartan de estos paradigmas se acusan de ser acientíficas y de no poseer una estructura teórica que los integre en forma coherente; tratando de negar las contradicciones existentes, ocultando la verdadera esencia de los problemas.

Por ello, es necesario develar la existencia de un componente ideológico que ha utilizado este paradigma para sus fines, bajo un interés puramente de carácter técnico. Interés técnico que orienta, hoy día, a la ciencia natural y a la tecnología las cuales son utilizadas para legitimar en la práctica relaciones de dominación, colocando en tela de juicio a las operaciones analíticas de tipo neutral que limitan el acceso al conocimiento de lo humano (Habermas, 1997).

Esto no implica desconocer el papel que ha jugado en el conocimiento de lo social y educativo el paradigma empírico analítico, de lo que se trata es de reafirmar que lo social no sólo es una realidad objetiva interpretable desde lo cuantitativo; que el mundo de lo humano no es abstracto, sino que constituye una realidad concreta que en esencia es un mundo de vida intersubjetivo valorable en lo cualitativo.

\section{TORNO A LAS PROPUESTAS CUALITATIVAS}

La investigación cualitativa, de carácter crítico, surge como un reto a las explicaciones positivistas dadas por la ciencia. En educación, se presentan como alternativas que van más allá de lo establecido, en cuanto a teorías y metodologías se refiere; con bases filosóficas y fundamentos epistemológicos que dan un giro humanista a la visión de la realidad social y educativa, al ser ahora objeto de múltiples interpretaciones que se caracterizan de acuerdo a 
un enfoque particular, ya sea reflexivo, crítico o interpretativo, desde una perspectiva microsocial.

El currículo educativo, entendido como una entidad concreta para la formación, como un proyecto de vida educativo y social viene a constituirse en el espacio dinámico escolar formal que da forma y sienta las bases para la realización de prácticas educativas e investigativas más humanas y vivenciales, entendidas éstas como actos sustantivos y comprometidos que recrean lo cognitivo y lo valorativo de los actores participantes (Delgado, 2001).

\section{LA INVESTIGACIÓN EDUCATIVA COMO PRÁCTICA DE SIGNIFICACIÓN}

Hoy día, como dice Colom (2004), se vive una subversión paradigmática, expresada por la emergencia de reivindicar al ser humano como sujeto pensante, actuante, creador y constructor de su vida y de la vida social total, frente al sometimiento a un mundo que lo margina como persona y lo reconoce como objeto eficiente y capaz de ejecutar una técnica y producir bienes económicos. Esta emergencia se afianza por la necesidad de la comprensión cualitativa del sujeto con sus pensamientos y creencias; afectos, actitudes y valores; prácticas, productos simbólicos y significados como constructor de su existencia.

Las ciencias sociales y educativas se encuentran, hoy día, en esta disyuntiva histórica: por una parte el agotamiento de la hegemonía del paradigma positivista, y por la otra, el surgimiento de paradigmas en los cuales lo cualitativo y lo humano, con todo lo que ello supone, reclaman su espacio vital.

La crítica por la deshumanización, consecuente producto de los paradigmas vigentes, ha llevado a un cuestionamiento de sus postulados e ideologías dominantes. Esta apreciación es válida, para los sistemas imperantes, tanto el capitalismo con el paradigma empírico analítico y positivista, y el socialismo marxista con el paradigma crítico radical.

El primero, con una visión de la persona y de la sociedad desde la razón técnica con la explicación de lo humano a través de las metodologías de las ciencias naturales; y el segundo, contrario al primero, con una visión materialista y estructuralista de la sociedad 
fundada en la crítica radical al capitalismo, pretende la ruptura de las estructuras sociales en el plano de lo material (Colom, 2004).

En lo educativo, éstos paradigmas han conformado la estructura del currículum educativo tradicional, en el que la teoría educativa y la práctica de investigación, presentan características particulares, así lo plantea Da Silva al expresar: “...una visión tecnicista resaltando las dimensiones instrumentales, utilitarias y económicas de la educación y del currículo, y una visión crítica marxista basada en un análisis crítico materialista de la escuela, de la educación y del conocimiento" (1998, p. 63).

Desde otras posturas, en tanto, se postula el retorno del sujeto, la valorización de las prácticas cotidianas particulares y el entendimiento de lo humano desde la intersubjetividad y la interdisciplinariedad,

...con el renovado paradigma crítico, que retoma y reformula algunas de las premisas del análisis crítico neomarxista, resaltando el currículum y la investigación como prácticas sociales críticas en continuo movimiento dialéctico. $\mathrm{Y}$, el fenomenológico crítico con la interpretación de la sociedad a partir de representaciones, simbolismos y significados de los discursos, textos y prácticas cotidianas. Conformando múltiples escenarios de redes intrincadas que deben ser visualizadas como espacios multiculturales e interdisciplinarios. (Colom, 2004, p. 64)

En el mismo sentido, Morin analiza lo social y educativo desde la complejidad, ...el conocimiento de la vida es el conocimiento de la vida misma. La frontera que nos separa de los otros seres vivientes no es una frontera natural: es una frontera cultural, que no anula la vida sino que la abre al desarrollo del espíritu expresado en lenguajes.... Penetrar la madeja de la vida en sus inconmensurables dimensiones exige de un método basado en la interdisciplinariedad y la multiculturalidad, para encontrar la unidad de la diversidad... (2002, p. 67)

Posturas metodológicas que tienen gran impacto en la educación, la cual debe constituirse en el espacio vital donde deben producirse los cambios requeridos, a través de un currículum con un espíritu humanista, que por su mismo carácter interdisciplinario conjugue las diversas expresiones del saber y el abordaje de los nuevos conocimientos. Cambios que 
exigen el encuentro de todos los saberes que componen el espacio educativo, hoy renovados y con propuestas coincidentes en torno al ser humano.

En lo cultural social, estos cambios conciben al ser humano, capaz de pensamiento y acción, con un grado de madurez, considerada como madurez histórica (Rizales, 2003), que se explica como un proceso de construcción de significados con sentido en el marco de las realidades naturales, culturales, económicas, políticas y sociales, que constituyen la suma de experiencias del mundo de la vida que le dan una conformación particular al sujeto a medida que crece como persona.

Postulados que revitalizan el concepto del ser humano como sujeto concreto, concebido en la unidualidad: natural-cultural, cerebral-psíquica, material-espiritual (Morin), en la que vive consustanciado con la naturaleza, con los demás, y consigo mismo, formando un todo indivisible; mujeres y hombres como hacedores de su mundo histórico cultural-social y del escenario de prácticas de significación, mediante la actuación activa, consciente y crítica, reflejada en una concreción personal-social que nunca estará completamente acabada.

Estas perspectivas revelan concepciones de la vida y de la cultura que no pueden quedar al margen de los proyectos educativos y de las prácticas investigativas, entendidos éstos como escenarios de recreación del conocimiento con sus tramas de significados expresados en acciones, discursos y textos particulares.

Por estas razones, se reafirma que el ser de la educación se construye y reconstruye subjetivamente en el encuentro intersubjetivo con los otros en lo escolar y social, entendidos éstos como escenarios dinámicos productores de prácticas de significación, en los que la investigación cumple un papel primordial.

En consecuencia, mediante el currículum construimos nuestra posición de sujeto y nuestra posición social, la identidad cultural y social de nuestro grupo. Producimos significados y sentidos que queremos que prevalezcan relativamente en los significados y sentidos de otros individuos y grupos. 
Por tanto, la investigación educativa juega un papel decisivo en esta realización, ya que se constituye en el espacio fundamental para la construcción de significados culturales y educativos. En tanto, confluyen y se entrecruzan en ella prácticas de significación, de identidad social y de poder.

Por ello se comparte con Ávila, cuando postula:

la investigación social y educativa es una empresa ética y política para el conocimiento y la promoción del ser humano y de un ideal de sociedad donde se le satisfagan plenamente sus derechos humanos y espirituales y materiales todas sus necesidades vitales, individuales y colectivas, sociales, históricas. (2005, p. 71)

Se reafirma, de esta manera, la tesis: que currículum, teoría educativa y práctica investigativa deben ir de la mano para la construcción del ser humano y de la sociedad que se aspira a partir de la educación. El currículum educativo, por tanto, se constituye en el espacio de vida escolar e institucional que da forma y sienta las bases para la realización de prácticas educativas e investigativas cualitativas, entendidas éstas como actos sustantivos y comprometidos que recrean lo cognitivo y lo valorativo de los actores participantes.

\section{EN RELACIÓN AL PARADIGMA FENOMENOLÓGICO CRÍTICO}

El paradigma fenomenológico crítico constituye hoy día la visión compartida de un conjunto de concepciones y enfoques epistemológicos y metodológicos que tienen como punto de encuentro, el significado dado por los sujetos participantes a las acciones y sucesos que constituyen la trama de su vida cotidiana y la recuperación de lo cualitativo (Rusque, 1999).

En este paradigma también llamado interpretativo, comprensivo o hermenéutico, confluyen perspectivas metodológicas que tienen valores comunes e intereses centrales: La investigación orientada más hacia la reconciliación que al cambio, el sujeto entendido como un sujeto constructor de la vida social en su vida cotidiana; el estudio de las prácticas sociales cotidianas; la realidad social como expresión de procesos subjetivos e interactivos que se articulan a un sistema compartidos de significados y valores; los eventos culturales como formas simbólicas susceptibles de ser comprendidas e interpretadas; el diálogo, como la participación, al menos de dos entidades que comparten sentido en el lenguaje; el papel 
del investigador como observador y participante; la descripción como principio de la investigación y, el más importante por su definición en lo epistemológico, el entendimiento de cómo funciona la asignación de significados a la acción social.

Para la investigación, en el marco de este paradigma, los datos de la experiencia vivida parten de lo real, de lo interno de cada individuo, recuperando la vida misma de los colectivos en lo discursivo y reflexivo como elementos fundamentales para las metodologías cualitativas, expresiones de la creación y recreación dinámica de la realidad social.

Se busca comprender, mediante la investigación, los significados de los actores con respecto a procesos sociales particulares, profundizar en el conocimiento de la forma cómo se experimenta la vida social a partir de describir los diversos contextos y situaciones; identificar aspectos relativos a los valores, las motivaciones, y las acciones que se manifiestan en las prácticas colectivas. Relacionar e interpretar los modos de hacer con sus diferentes significados en el contexto situacional en el cual se producen.

Es así como, el paradigma fenomenológico abre un camino metodológico a las ciencias culturales, históricas y sociales. Para los fines de la investigación se trata de ir a la vivencia original de la experiencia propiamente dicha, lo vivido, lo real, lo interno, en lo discursivo y reflexivo como elementos fundamentales de las metodologías cualitativas; describiendo así los elementos que constituyen los saberes humanos, apoyándose en algo implícito, formulado tal como es vivido.

En la investigación educativa, los actores del proceso educativo no actúan simplemente en los hechos sociales, sino que son ellos los que están "creando", por decirlo así, sus propios mundos sociales en interacción con los otros y que para ello requieren una metodología especial.

Cada individualidad se comprende como un todo estructurado, una red de conexiones que no puede atomizarse; es, por tanto, la representación de su mundo cultural - social, con sus valores y significados, los que intercambia, deconstruye, construye y reconstruye con los otros en el encuentro para lo educativo. Los valores, aspiraciones o ideales, que cada individuo tiene imprime a las acciones colectivas un sentido que se constituye en la suma de 
lo social en el contexto escolar, expresiones de la creación y recreación dinámica de la realidad social.

Se destaca, por tanto, que existe en la actualidad la necesidad de volver al sujeto, profundizar en el conocimiento de lo humano en todas sus manifestaciones, cuestiones que involucran lo subjetivo, lo valorativo y representativo de lo cultural social. Panorama que ofrece las perspectivas cualitativas con la revalorización de la experiencia vivida en términos del significado que tiene para los diferentes actores.

\section{LOS INTERESES DE LA INVESTIGACIÓN CUALITATIVA}

El interés central de la perspectiva cualitativa en la investigación social y por ende, en la investigación educativa se expresa en: el sujeto, la subjetividad, los significados y los espacios de mediación simbólica. Para Morin, la subjetividad ha entrado por la puerta grande del pensamiento social, en el sentido que este paradigma incorpora muchos de los asuntos vinculados con la actividad del actor; una subjetividad, en primer lugar, que se refiere a la naturaleza misma del sujeto, aparece en el cómo el sujeto construye los significados. Y, en segundo lugar, la subjetividad implícita en los procesos interactivos, en el sistema compartido de símbolos, en los significados de la acción, en las definiciones subjetivas de la situación, en las mediaciones sociales, el lenguaje, la comunicación y la cultura, elementos que conforman una subjetividad objetivizante que nace de la relación compleja que se establece con los otros (Rusque).

La intersubjetividad en lo socioeducativo se genera del intercambio cotidiano de la interacción escolar y social. En esa interacción se negocian entre los distintos actores las características constitutivas de la realidad para el colectivo o grupo. Es así como, se le devuelve el lugar al sujeto en la vida cotidiana, la vida de todos los días, que nos permite sentirnos sujetos y ver a los otros también como sujetos.

Para la investigación social y educativa, el sujeto que interesa es el sujeto en relación al mundo donde vive, mundo producido y construido por él; un mundo lleno de significados que se relaciona con las otras personas. Está mediado por normas, por reglas del lenguaje ordinario. En otras palabras, durante su vida el sujeto ha ido descubriendo los significados de Volumen 8, Número 3, Año 2008, ISSN 1409-4703 
los significados recibidos, a la vez que ha llenado los significados recibidos con su experiencia personal, lo que constituye un manojo de posibilidades abiertas e indeterminadas para la investigación educativa.

Los significados se constituyen en la naturaleza del objeto de la investigación. Estos significados son productos sociales en el sentido que son formados o transformados por el proceso de definición que tiene lugar en la interacción. Las personas están preparadas a actuar hacia los objetos sobre la base de los significados que dichos objetos tienen para ellos. Como otro de los procesos que realizan los sujetos, la asignación de significados corresponde a atribuciones que ellos hacen sobre los objetos, ya que éstos no tienen existencia propia, ni naturaleza intrínseca. Un hecho que se da mediante la objetivación a través del cual se le atribuyen a un objeto determinadas propiedades que son válidas en determinados ámbitos específicos.

Los espacios de mediación simbólica se encuentran constituidos por el lenguaje, la familia, los grupos primarios, la escuela, en general, por los factores de la cultura que actúan como componentes objetivados y subjetivados que necesitan ser explicitados en términos analíticos para poder comprender la vida social en lo particular y total, lográndose una mejor comprensión de las relaciones entre individuo, sociedad y escuela, tanto a través de factores y espacios mediadores como de mediación, donde se establecen las complejas relaciones entre la acción humana y el contexto cultural social.

El sujeto de la educación se mueve en hechos y contextos interactivos, dinámicos y complejos, donde se producen relaciones cara a cara, recreándose en cada encuentro la realidad social y educativa. En este medio, el sujeto capta el sentido de la realidad social, la comprensión de sí mismo y de la realidad socioeducativa. La interacción social y educativa, por tanto, se fundamenta en la apertura de los sujetos a la comunicación y a la negociación de los significados.

\section{LA RELACIÓN SUJETO-OBJETO Y EL PAPEL DEL INVESTIGADOR}

Las perspectivas cualitativas privilegian y promueven la proximidad entre el investigador y el objeto de investigación (el sujeto, si se trata de personas), al exigir la presencia del Volumen 8, Número 3, Año 2008, ISSN 1409-4703 
investigador a través de la observación participante u otras técnicas de recolección de información en la búsqueda del conocimiento. Lo que requiere del uso de un recurso metodológico de perspectivas cualitativas que justifique la presencia prolongada e intensa del investigador y demás actores participantes en el proceso y, en el escenario de la investigación; perspectivas cualitativas que proponen un cambio al reunir el objeto y reconstruir el sujeto que concibe al objeto, en un proceso constitutivo mutuo, tal como lo plantea la fenomenología.

Necesariamente esta situación remite al problema filosófico de la relación sujeto-objeto, pero no a partir de la especulación exterior de la ciencia sino del desarrollo interno, en la práctica, de un principio de complejidad donde podamos reconocer al objeto, viéndolo como un par: sujeto/objeto. Es el sujeto quien emerge del objeto, por decirlo así, y revierte sobre el observador, sobre el investigador, incitándolo a interrogarse y a incluirse en su propia investigación (Morin). En este proceso, el sujeto prácticamente se acepta como observador en una relación de incertidumbre con el sujeto, en la medida que asume la postura radical de una relación abierta sujeto-objeto.

Según el autor antes citado, al paradigma de la diferenciación entre sujeto-objeto le sucede un paradigma de la incorporación del sujeto-objeto. Ha terminado la concepción del sujeto puro y del objeto puro. La objetividad y la subjetividad existen pero de manera relativa. La objetividad y la subjetividad existen la una y la otra de manera relacional, lo que plantea una nueva dimensión de la investigación en las ciencias sociales, donde no sólo hay una renovación del aparato conceptual y metodológico sino que también hay una reorientación de los procedimientos de observación y del análisis de los datos. Así se deja de lado algunas restricciones impuestas por el método positivista como es el principio de objetivación y de neutralidad axiológica: el sujeto se reintegra a la ciencia, a la naturaleza, a la vida, a la cultura, a la sociedad y deja de estar fuera, por debajo, por arriba en otra parte. Ya no se trata sólo del sujeto que reflexiona al objeto, o del objeto que reflexiona al sujeto, sino de un doble reflejo, una doble constitución, una organización bipolarizada.).

La tarea del investigador, por tanto, se asume en los términos anteriores dejando ver las contradicciones que pueden existir entre las interpretaciones dadas por el investigador y las de los otros actores del proceso. Esto hace que la verificación de hipótesis aparezca como 
un componente del trabajo de investigación que puede realizarse durante la etapa de recolección de datos o posteriormente.

En relación a lo anterior, se infiere que el investigador frecuentemente lleva al terreno de la investigación un gran contenido teórico, pero pocas ideas precisas sobre lo que va a suceder. Por ello utiliza, en principio, la observación participante combinada con entrevistas, y de esta manera establece una relación cara a cara con los actores, compartiendo su vida cotidiana. Las numerosas interrelaciones así producidas permiten que se generen hipótesis emergentes y los datos aumentan las posibilidades de formular nuevas hipótesis, por el descubrimiento de diferencias entre los datos esperados y los datos recogidos.

Rusque señala también que de esta manera la validez de los estudios que consideran al individuo o a la cultura humana, pasa por una interacción personal de larga duración entre el investigador y los sujetos. Es así como la duración de la observación se considera un factor de validación de una investigación cualitativa sobre el terreno, factor que está asociado a otro factor: la proximidad (interacción personal) entre el investigador y su medio.

La observación participante puede tomar un carácter más o menos activo o pasivo, según su nivel de implicación en los sucesos que observa y en los puntos de vista de los actores. El cuadro teórico y los problemas de investigación orientarán la toma de decisiones relativas a los tipos de implicación que el investigador debe tener con el medio. Por otra parte, la observación participante puede variar en intensidad y duración, constituyendo una técnica de observación adecuada para que el investigador pueda enfrentar la complejidad de la red de relaciones lingüísticas dentro de un contexto convencional, entendida la técnica como artefacto, nunca neutral.

De esta manera, en la investigación cualitativa, el investigador es el lugar donde la información se convierte en significación y en sentido, dado que la unidad del proceso de investigación en última instancia no está ni en la teoría ni en la técnica, ni en la articulación de ambas, sino en el investigador mismo. 


\section{ALGUNAS REFLEXIONES FINALES}

Frente a la crisis actual del paradigma establecido en las ciencias sociales, propuestas teóricas y metodológicas alternativas emergen desde diferentes posiciones con una perspectiva interdisciplinaria, bajo una visión humanista y cualitativa de lo educativo. Estas se inscriben en lo fenomenológico y crítico, con postulados que indican la urgencia del entendimiento de lo social desde lo humano; paradigmas que revitalizan a las ciencias sociales y auguran nuevos tiempos para la investigación educativa. Se busca la unidad de la diversidad, con el encuentro de los diferentes postulados epistemológicos y metodológicos y sus prácticas, tal como lo expresa Rusque:

...las ciencias sociales viven una etapa de reflexiones, una búsqueda de puntos comunes, cooperación, suplementariedad y complementariedad de los paradigmas que permitan el trabajo conjunto, dejando las posturas de rechazo mutuo.....esto no significa obviar las diferencias, se trata de plantearlas con la suficiente claridad para descubrir las fortalezas, dificultades y alcances de cada enfoque en el estudio de lo social, atenuando la rivalidad epistemológica entre el par cuantitativo - cualitativo, buscando su coexistencia en la práctica, que de acuerdo a la realidad a abordar tendrán un mayor o menor peso.... pero, manteniendo como una constante que se está investigando lo humano en sus diversas realidades. (1999, p. 98)

Esto indica, que debe privar en el investigador de las ciencias sociales y particularmente, en el investigador de lo educativo, la comprensión de lo social y educativo como realidades humanas que constituyen entramados culturales en lo histórico y social.

En este sentido, la investigación educativa como una categoría de la investigación en ciencias sociales comprendida desde la perspectiva cualitativa, se entiende como un acto sustantivo y comprometido que tiene el propósito de abordar las problemáticas y situaciones que afectan un contexto socioeducativo particular, con el fin de producir o descubrir nuevos elementos teóricos y realizar las acciones, que modifiquen o transformen la realidad estudiada, desde lo cognitivo y valorativo de las prácticas cotidianas. Lo que implica la generación de nuevos aprendizajes, nuevas teorías, reflexiones y acciones a partir de la investigación. 
Así, se requiere de la investigación educativa el cumplimiento del papel que le corresponde para la identificación y comprensión de aquellos aspectos del contexto socioeducativo que afectan y frustran los fines educacionales, y poder ofrecer interpretaciones teóricas mediante las cuales los actores involucrados eliminen o superen tales limitantes, reflexionando sobre sus propias situaciones, recreándolas y transformándolas a partir de sus propias acciones. Por tanto, la misión de la investigación educativa consiste en generar teorías sustantivas que tengan su fundamento en las complejidades de la realidad práctica.

La crítica al marcado sello positivista de las ciencias sociales tradicionales se manifiesta con mayor virulencia en la investigación social y, en particular, en la investigación educativa, debido a la urgente necesidad de reivindicar al sujeto como persona humana y el requerimiento expreso del uso de métodos cualitativos para su comprensión en el marco de la realidad histórico cultural social concreta y compleja donde éste se ubica.

Los nuevos tiempos van conformando nuevos escenarios humanos y socioeducativos, con situaciones complejas que por sus mismas características no pueden ser ya estudiadas con las metodologías tradicionales. Es decir, se destaca la presencia de nuevos escenarios y problemas que requieren de otras metodologías para abordarlos, interpretarlos y comprenderlos. De allí, que el reclamo actual, parta de la necesidad de recrear una concepción epistemológica y teórica basada en lo cualitativo que comprenda al sujeto en todas sus dimensiones de existencia, como un sujeto que piensa, siente, interpreta, planifica, elige y actúa.

Por las razones antes expuestas, se requiere, en primer lugar, reconocer que existe un componente ideológico politico que subyace en la investigación social y por tanto, en la investigación educativa; que investigar en educación exige de una toma de posición ante las problemáticas; que las problemáticas deben ser abordadas desde un plano crítico y comprometido; que el investigador educativo no puede ser un objeto para la reproducción sino el sujeto que busca la transformación; que lo humano en la dimensión de las prácticas sociales no puede ser generalizable, ya que cada práctica es una manifestación de carácter particular en lo cultural y cada acción humana lleva una intencionalidad que se entiende en el plano de lo subjetivo y por los significados que los actores le otorgan a sus acciones. 
Declarar la existencia de un componente ideológico-político en la investigación educativa pasa por reconocer también que no existe neutralidad científica, ni en el modo de producción del conocimiento, ni en los resultados que de él derivan. Esto abre la posibilidad para la utilización de métodos alternativos de investigación, ya que lo fundamental no es el método en sí, sino el problema planteado (Rusque). El método sigue al problema y nunca viceversa, las investigaciones deben entonces evaluarse en función de sus efectos sobre la realidad y no de las declaraciones explícitas de sus realizadores.

En tal sentido, la realidad social y la realidad educativa se entienden como construcciones cotidianas que deben ser estudiadas en una perspectiva dialéctica en la que la educación y la sociedad se definen como mutuas construcciones que se transforman la una a la otra en la interacción continua y, en este proceso el sujeto humano como constructor y productor de su mundo elabora, reelabora y desecha cada día construcciones subjetivas de ese mundo. Estas elaboraciones, que son de carácter simbólico, se expresan mediante el lenguaje, en la comunicación que se da en la intersubjetividad, producto del encuentro con los otros.

Es por esto, que al volver a la concepción positivista de la sociedad y de la educación, encontramos que los fenómenos de alienación presentes en la concepción positivista de la investigación educativa han bloqueado la capacidad de comunicación y de producción de conocimientos, debido a que en este paradigma la teoría se establece previamente y el producto de la investigación se ajusta a los criterios predeterminados.

Mientras que en la perspectiva cualitativa, la teoría se utiliza para iluminar, clarificar, no se utiliza para prescribir, sino para enriquecer la interpretación, no constituye una explicación previa de lo que se encontrará en la realidad, sino una ayuda para la indagación, susceptible de ser rebatida por la singularidad de los procesos (Popkewitz, citado por Elliott, 1998).

Por lo anterior, se plantea el carácter esencialmente activo de los sujetos de la investigación educativa, ya que como constructores y actores de su propia realidad tienen derecho a participar en el proceso investigativo y a conocer los resultados del mismo para llevar a cabo los cambios y transformaciones requeridas. La relación entre investigador e investigados se convierte en una relación horizontal de encuentro dialógico en la que ambos comparten conocimientos. Se incorpora así la concepción dinámica, compleja y dialéctica de los seres 
humanos y de su interacción, no vista ya como elemento a estudiar, sino como característica esencial de toda actividad humana.

En consecuencia, el interés esencial del cambio y transformación social y educativa en la actualidad, más que nunca, debe estar revestida de significación social; debe abandonarse el culto a los métodos tradicionales desarrollados por las ciencias naturales, según los dictámenes del paradigma dominante, y recrear alternativas que den respuestas a los problemas socioeducativos, propósito de la investigación educativa.

\section{REFERENCIAS}

Ávila, Renee. (2005). Investigación Educativa y cambio social. Bogotá: Editorial ágora.

Colom, Dan. (2004, 4 de mayo). El papel de la investigación educativa en un mundo cambiante. Conferencia dictada en el Congreso de Gerencia Educativa NURRULA, Cartagena.

Da Silva, Tomáz. (1998). Cultura y Currículo. Barcelona, España: Editorial Gedisa.

Delgado, Flor. (2006). Paradigmas y Retos de la Investigación Educativa. Una Aproximación Crítica. Venezuela: Consejo de Publicaciones de la Universidad de Los Andes.

Esqueda, Carlos. (2004). El Retorno Cualitativo en las Ciencias Humanas. Venezuela: Editorial, Prensa Escrita, Unir.

Habermas, Jurgen. (1997). Teoría Crítica de la Sociedad. Madrid: Editorial Tecnos.

Morín, Edgar. (2002). La Educación del Futuro (Traducción de A. Rota, Bogotá). Paris: UNESCO.

Rizales, Alfredo. (2004). La educación y sus retos en el siglo XXI. Caracas: Editorial Gafrica.

Rusque, Ana María. (1999). De la Diversidad a la Unidad en la Investigación Cualitativa. Caracas: FACES-UCV. 